\title{
E2FI induces LSINCT5 transcriptional activity and promotes gastric cancer progression by affecting the epithelial-mesenchymal transition
}

This article was published in the following Dove Press journal:

Cancer Management and Research

\author{
Peng Qi, ${ }^{1-3}$ \\ Wan-run Lin, ${ }^{4}$ \\ Meng Zhang, ${ }^{1-3}$ \\ Dan Huang, ${ }^{1-3}$ \\ Shu-juan $\mathrm{Ni}^{1-3}$ \\ Xiao-li Zhu, ${ }^{1-3}$ \\ Qian-ming Bai, ${ }^{1-3}$ \\ Wei-qi Sheng, ${ }^{1-3}$ \\ Xiang Du, ${ }^{2}$ \\ Xiao-yan Zhou ${ }^{1-3}$ \\ 'Department of Pathology, Fudan \\ University Shanghai Cancer \\ Center, Shanghai, China; ${ }^{2}$ Institute \\ of Pathology, Fudan University, \\ Shanghai, China; ${ }^{3}$ Department of \\ Oncology, Shanghai Medical College, \\ Fudan University, Shanghai, China; \\ ${ }^{4}$ Department of Pathology, University \\ of Texas Southwestern Medical \\ Center, Dallas, TX, USA
}

Correspondence: Xiao-yan Zhou Department of Pathology, Fudan University Shanghai Cancer Center, 270 Dong An Road, Shanghai 200032, China

Tel +862164175590 ext 8330

Fax +862164170067

Email 13524324387@163.com
Background: Long noncoding RNAs (lncRNAs) have been shown to play important regulatory roles in human cancer. We previously verified that the lncRNA long stress-induced noncoding transcript 5 (LSINCT5) is overexpressed in gastric cancer (GC) cells and closely correlated with cell proliferation and patient prognosis. However, whether aberrant LSINCT5 expression has an important effect on GC progression is unclear, and the potential mechanisms remain unknown. In GC, E2F1 expression is also aberrant, but the biological functions of E2F1 are controversial, and the correlation between E2F1 and lncRNAs remains unknown.

Materials and methods: Expression of LSINCT5 was analyzed in metastatic GC tissues compared with nonmetastatic tissues using quantitative real-time PCR (qRT-PCR) assays. Gain and loss of function approaches were used to investigate the biological role of LSINCT5 in GC cell migration and invasion. A computational screen of LSINCT5 promoter was conducted to search for transcription factor-binding sites. LSINCT5 promoter activities were examined by ChIP and luciferase reporter assays. qRT-PCR and western blotting assays were performed to detect the expression of multiple EMT markers in cells in which LSINCT5 was overexpressed or knocked down.

Results: An integrated quantitative analysis revealed that LSINCT5 was significantly overexpressed in metastatic GC tissues. Forced LSINCT5 expression promoted cell migration and invasion, whereas loss of LSINCT5 function decreased cell migration and invasion. Mechanistic investigations showed that LSINCT5 is a direct transcriptional target of E2F1. Moreover, LSINCT5 overexpression was found to play an important role in the epithelial-to-mesenchymal transition by regulating the expression of E-cadherin, $\mathrm{N}$-cadherin, vimentin, and matrix metalloproteinase-2. Conclusion: These data suggest that E2F1-mediated activation of LSINCT5, a regulator of cell migration and invasion, constitute the mechanistic link between the E2F1-mediated pathway and lncRNA that regulates cell migration and invasion. Thus, LSINCT5 may be a target for new GC therapies.

Keywords: E2F1, LSINCT5, metastasis, gastric cancer

\section{Introduction}

Gastric cancer (GC) is one of the major health problems in the world. ${ }^{1}$ Although the global mortality rates from GC have decreased over the past several decades, mortality remains extremely high in many countries, where many patients are diagnosed with advanced stage disease and have very poor survival. ${ }^{2}$ However, traditional prognostic assessments based on histological differentiation and the TNM stage are unable to indicate a tumor's metastatic status or predict the clinical outcome of a patient. As a result, there is a growing need to explore novel prognostic biomarkers for assessing the metastasis process. 
The key role of the epithelial-mesenchymal transition (EMT) in cancer cell metastasis was elucidated in the past decade. ${ }^{3}$ This biological process is characterized by the loss of epithelial markers and the acquisition of mesenchymal markers, and E-cadherin, N-cadherin, and vimentin are considered the most important molecular markers of the EMT. More recently, several studies have implicated long noncoding RNAs (lncRNAs), which are key RNA molecules $>200$ nucleotides in length, in tumorigenesis, cancer progression, and metastasis. ${ }^{4,5}$ For instance, our research groups have demonstrated that PVT1 enhances GC cell proliferation and invasion in a FOXM1mediated manner. ${ }^{6}$ Moreover, the lncRNA ncRAN mediates colorectal cancer (CRC) cell migration and invasion and might be a novel prognostic indicator, specifically, a biomarker, for the early detection of metastasis in CRC.

Although a small number of lncRNAs have been functionally characterized, little is known regarding most lncRNAs. ${ }^{7}$ Long stress-induced noncoding transcript 5 (LSINCT5), which is $\sim 2.6-\mathrm{kb}$ long, is an intergenic lncRNA located at Chr $5 p$. Previous experiments showed that LSINCT5 expression was 7- and 10-fold higher in primary breast tumor tissues and breast cancer cell lines than in corresponding normal benign breast tissue and normal cell lines, respectively, and that the knockdown of LSINCT5 with antisense oligos decreased the proliferation of breast cancer cell lines. ${ }^{8}$ In our previous study, we verified that LSINCT5 is overexpressed in GC cells and closely related to cell proliferation and patient prognosis. ${ }^{9}$ However, whether aberrant LSINCT5 expression exerts an important effect on GC progression remains unclear, and the potential mechanisms are unknown.

E2F transcription factors are key participants in numerous cellular events, such as the control of the cell cycle, DNA synthesis, and nuclear transcription. In mammals, 8 members of the E2F family have been identified, and these are divided into 4 groups based on homology: E2F1 to 3, E2F4 and 5, E2F6, E2F7 and 8 . The transcriptional activity of E2Fs is predominantly regulated by the $\mathrm{Rb}$ protein family ( $\mathrm{Rb}, \mathrm{p} 107$ and $\mathrm{p} 130),{ }^{10,11}$ and the activation of E2F1 to 3 is central to the regulation of cell cycle-related genes. ${ }^{12} \mathrm{E} 2 \mathrm{~F} 1$ is the most thoroughly investigated member of the E2F family with respect to human malignancies and plays pivotal roles in tumor progression by modulating the expression of both coding and noncoding transcripts. ${ }^{13,14}$ Interestingly, E2F1 has been reported to act as either an oncogene or a tumor suppressor to modulate tumorigenesis depending on the cell context. ${ }^{13,15,16}$ Emerging evidence has revealed that E2F1 has an important effect on GC progression, but its biological functions remain debated. ${ }^{17-19}$

Here, we demonstrate a novel pathway involving E2F1 and LSINCT5 in GC cell progression. In this study, we found the following: 1) LSINCT5 is overexpressed in metastatic GC tissues and has a significant effect on GC cell migration and invasion in vitro; 2) E2F1 induces the transcriptional activation of LSINCT5; and 3) LSINCT5 forces cancer progression by affecting EMT. This study advances our understanding of the role of IncRNAs, such as LSINCT5, as regulators of GC pathogenesis and facilitates the development of IncRNAdirected diagnostics and therapeutics.

\section{Materials and methods Plasmids and RNA interference}

The pMax-E2F1 expression vector was purchased from Addgene (plasmid \#16007) (Cambridge, MA, USA), and siRNAs specific for E2F1 (\#AM16708) and LSINCT5 (\#4392420) were chemically synthesized (Invitrogen, Carlsbad, CA, USA). For the construction of luciferase reporter vectors, the core promoter of the LSINCT5 gene $(-1,400$ to +100 relative to the transcription start site of the LSINCT5 gene) and regions containing binding site deletions were subcloned into the pGL3 basic firefly luciferase reporter. All the polymerase chain reaction (PCR) products were verified by DNA sequencing. Cell transfection was performed using Lipofectamine 2000 according to the manufacturer's instructions (Invitrogen).

\section{Cell lines}

The human gastric adenocarcinoma cancer cell lines MGC803, BGC823, MKN45, AGS, and SGC7901, and the normal gastric epithelium cell line GES-1 were obtained from the Chinese Academy of Sciences Committee on Type Culture Collection Cell Bank (Shanghai, China). The cells were cultured in Roswell Park Memorial Institute 1640 medium (Invitrogen) or DMEM (HyClone, Logan, UT, USA). All media were supplemented with $10 \%$ fetal bovine serum (FBS) and $2 \mathrm{mM}$ L-glutamine (Invitrogen), and all the cells were cultured at $37^{\circ} \mathrm{C}$ in a $10 \% \mathrm{CO}_{2}$ atmosphere.

\section{Tissue samples and clinical data collection}

In this study, 71 patients underwent primary GC resection at the Fudan University Shanghai Cancer Center. ${ }^{9}$ The study was approved by the Ethics Committee on Human Research of the Fudan University Shanghai Cancer Center. Written informed consent was obtained from all the patients.

\section{RNA preparation and quantitative real- time PCR (qRT-PCR)}

Total RNA was extracted with the TRIzol reagent (Invitrogen, Grand Island, NY, USA), and qRT-PCR analyses were 
conducted according to the manufacturer's instructions (Takara, Dalian, China). The results were normalized to the expression of glyceraldehyde-3-phosphate dehydrogenase (GAPDH) using the comparative $2^{-\Delta \Delta \mathrm{Ct}}$ method. Each sample was assayed in triplicate, and the primer sequences are listed in Table S1.

\section{Luciferase reporter assay}

Cells were transfected with appropriate plasmids in 24-well plates for 48 hours, collected, and lysed for luciferase assays. The relative luciferase activity was obtained by normalizing the activity of firefly luciferase to that of Renilla luciferase.

\section{Wound healing assay}

The wound healing assay was used to evaluate the tumor cell motility capacity. Briefly, $3 \times 10^{5}$ cells were seeded in 6-well plates, cultured overnight, and transfected with the control, pcDNA-LSINCT5, ${ }^{9}$ or si-LSINCT5. When the cells reached $\sim 85 \%$ confluence, the cell layer was scratched with a sterile plastic tip, washed twice, and cultured in complete medium. Wound gaps were observed for 48 hours, and images were captured every 24 hours at $100 \times$ magnification, then the migration rate was calculated accordingly. The data were summarized based on 3 independent assays.

\section{Cell invasion and migration assay}

The upper sides of MilliCell (12-mm diameter with 8- $\mu \mathrm{m}$ pores) chambers (Millipore, Billerica, MA, USA) were pre-coated with Matrigel (BD Biosciences, Bedford, MA, USA). A total of $5 \times 10^{4}$ cells in serum-free medium were added to the upper compartment in medium supplemented with $0.1 \%$ FBS, and the chambers were placed into 24 -well plates with medium containing $10 \%$ FBS. After incubation for an appropriate time at $37^{\circ} \mathrm{C}$ in $5 \% \mathrm{CO}_{2}$, the cells remaining on the upper membrane were removed with a cotton swab, and the invaded cells on the lower membrane surface were stained with $4 \%$ paraformaldehyde and crystal violet. The invasive activity was quantified by counting 9 random fields (at 200× magnification) per chamber. For each experimental point in each assay, mean values were obtained from at least 3 individual chambers. The migration assay was performed using the same protocol as that used for the invasion assay with the exceptions that no Matrigel was used and the cells were allowed to permeate for 12 hours.

\section{Chromatin immunoprecipitation (ChIP)} ChIP assays were performed using an EZ-ChIP ${ }^{\mathrm{TM}} \mathrm{Kit}$ (Millipore) according to the instruction manual. E2F1 antibody was obtained from Cell Signaling Technology (Danvers,
MA, USA). The immunoprecipitated DNA was quantified by qPCR using SYBR Green Master Mix (Takara). ChIP data are presented as percentages relative to the input DNA.

\section{Western blotting analysis}

Cells were collected and lysed using radioimmunoprecipitation assay protein extraction reagent (Beyotime, Beijing, China) supplemented with a protease inhibitor cocktail and phenylmethylsulfonyl fluoride (Roche, Pleasanton, CA, USA). The protein concentration was determined using a Bio-Rad protein assay kit (Bio-Rad, Hercules, CA, USA). Approximately 50 $\mu \mathrm{g}$ of protein extract was separated on $10 \%$ sodium dodecyl sulfate-polyacrylamide gel electrophoresis gels, transferred to nitrocellulose membranes (Sigma, St. Louis, MO, USA) and incubated with specific antibodies. Enhanced chemiluminescence chromogenic substrate was used to visualize the bands, and the band intensities were quantified by densitometry (Quantity One software; Bio-Rad) using GAPDH as the control. Antibodies (1:1,000 dilution) against E-cadherin and $\mathrm{N}$-cadherin were purchased from BD (BD Biosciences), and antibodies against vimentin, matrix metalloproteinase (MMP)-2 and MMP-9 were purchased from Cell Signaling Technology.

\section{Statistical analysis}

All data analyses were performed using SPSS statistical software (Version 20.0; SPSS Inc., Chicago, IL, USA). The significance of the differences between groups was evaluated using Student's $t$-test or chi-squared test, as appropriate. The Pearson correlation test was used to investigate the correlation between E2F1 and LSINCT5 mRNA expression. Two-sided $P$-values were calculated, and a statistical significance level of 0.05 was chosen.

\section{Results \\ LSINCT5 is overexpressed in metastatic GC tissues and exhibits a significant effect on GC cell migration and invasion}

Our first goal was to investigate whether LSINCT5 expression is altered in metastatic GC tissues compared with nonmetastatic tissues. Using GAPDH as the normalization control, we found that LSINCT5 expression was significantly higher in metastatic GC tissues than in nonmetastatic GC tissues (Figure 1A; $P<0.05$ ).

Because our previous study validated an association between LSINCT5 and GC cell growth, ${ }^{9}$ we further investigated the biological role of LSINCT5 in GC cell migration and invasion using gain- and loss-of-function approaches. LSINCT5 was overexpressed by transfecting MGC803 cell 


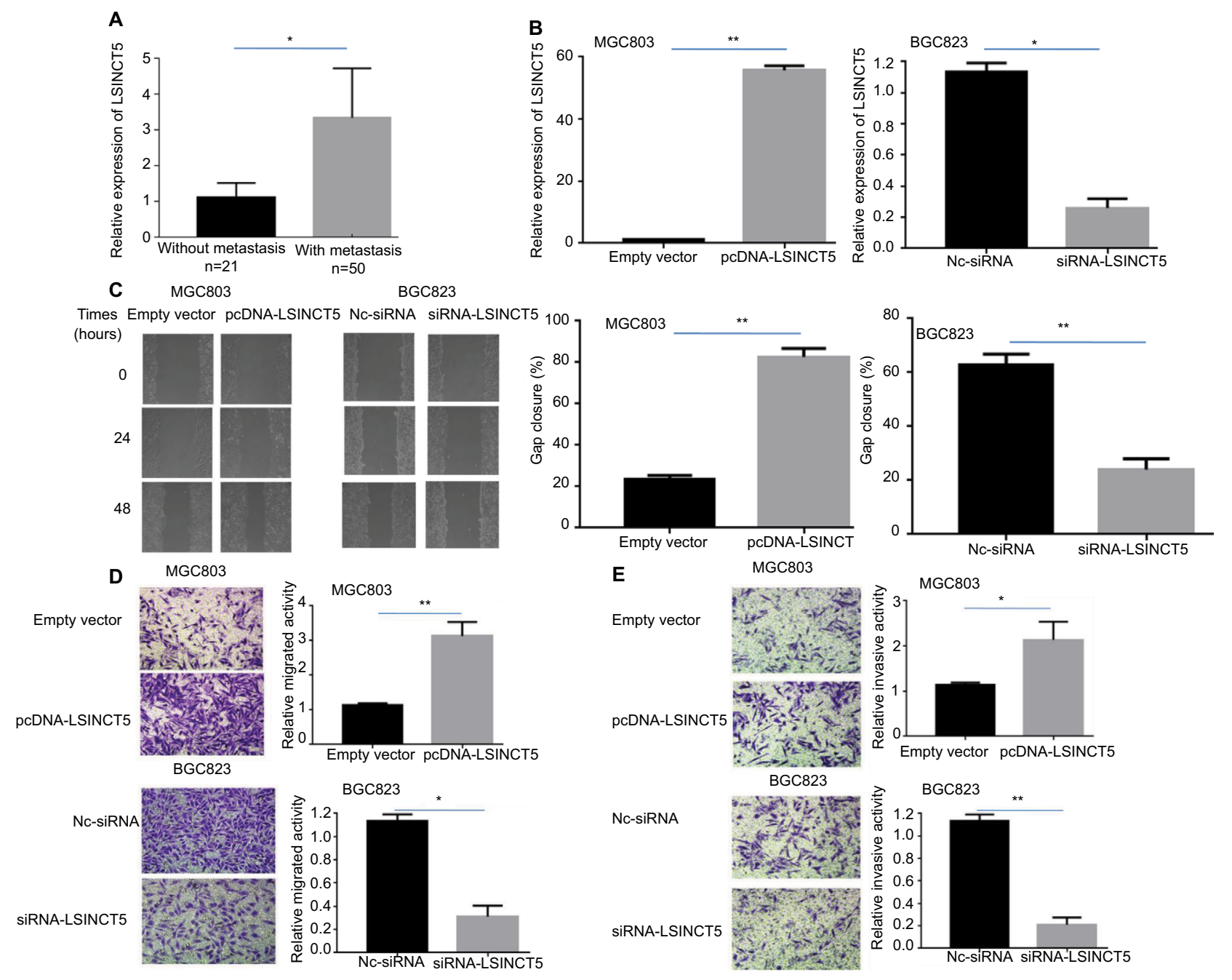

Figure I Effects of LSINCT5 on GC cell migration and invasion.

Notes: (A) qRT-PCR analysis of the LSINCT5 expression levels in metastatic and nonmetastatic GC tissues. (B) qRT-PCR analysis of the LSINCT5 expression levels following the transfection of MGC803 cells with empty vector or pcDNA-LSINCT5 (left panel) or following the transfection of BGC823 cells with scrambled siRNA or siLSINCT5 (right panel). (C) Wound healing assays were performed to investigate the migratory ability of GC cells. (D, E) Transwell assays were used to investigate changes in the migratory and invasive abilities of GC cells. All the experiments were performed in triplicate. Bars: $S D ; * P<0.05$ and $* * P<0.0$ I.

Abbreviations: GC, gastric cancer; qRT-PCR, quantitative real-time polymerase chain reaction; NC, negative control.

lines, which have relatively low LSINCT5 expression, with the pcDNA-LSINCT5 vector, and chemically synthesized small interfering RNAs (siRNAs) were used to knockdown LSINCT5 expression in BGC823 cells, which have relatively high LSINCT5 expression. The ectopic expression and depletion of LSINCT5 in these cells was confirmed by qRTPCR (Figure 1B). A wound healing assay showed that cell migration was promoted in pcDNA-LSINCT5-transfected MGC803 cells compared with control cells (Figure 1C), and BGC823 cells exhibited a slower scratch closure rate (inhibition of migration) after the knockdown of LSINCT5 than of control siRNA (Figure 1C). We then investigated the invasion and migration of GC cells through Matrigel and transwell assays, respectively. As shown in Figure 1D, E, MGC803 cell migration and invasion were promoted following the upregulation of LSINCT5 expression, and reduced LSINCT5 expression significantly hindered the migration and invasion of BGC823 cells compared with the control. These results suggest that LSINCT5 may be closely related to GC cell migration and invasion.

\section{E2FI upregulates LSINCT5 expression in GC cells}

To explore the role of E2F1 in human GC progression, we examined the E2F1 expression level in gastric tumor tissues (Ts) and paired adjacent normal tissues (ANTs) and found that the E2F1 mRNA levels were 3.2-fold higher in Ts than in ANTs (Figure 2A). In addition, we determined that E2F1 was more highly expressed in metastatic tumor tissues than in nonmetastatic tissues (Figure 2B). We next examined the 
E2F1 expression levels in 1 normal gastric epithelium cell line (GES-1) and in several GC cell lines and demonstrated that $\mathrm{E} 2 \mathrm{~F} 1$ expression was significantly higher in the GC cell lines than in the GES-1 cell line (Figure 2C).

Previous data have shown that E2F1 promotes cancer progression by activating the transcription of downstream target coding and noncoding genes. To ascertain whether LSINCT5 is regulated by E2F1 expression in GC, we performed a ChIP assay using E2F1 antibody and to quantify the immunoprecipitated DNA, and the results revealed that endogenous E2F1 could bind to the LSINCT5 promoter, in untreated GC cells or increased or reduced E2F1 expression
GC cells (Figure 2D). Then, we cloned the human LSINCT5 promoter fragment (nucleotides $-1,400$ to +100 ) into a pGL3 vector and performed luciferase activity assays and found that E2F1 overexpression induced LSINCT5 transcriptional activity (Figure 2E), which suggests that E2F1 participates in the transcriptional regulation of LSINCT5. To further confirm this notion, we examined the LSINCT5 core promoter region for transcription factor-binding sites using online software programs (Jaspar: http://jaspar.genereg. net/, ChIPBase: http://deepbase.sysu.edu.cn/chipbase/, and TFSEARCH: $\underline{\text { http://www. cbrc.jp/research/db/TFSEARCH. }}$ html) and identified four predicted tandem E2F1-binding sites
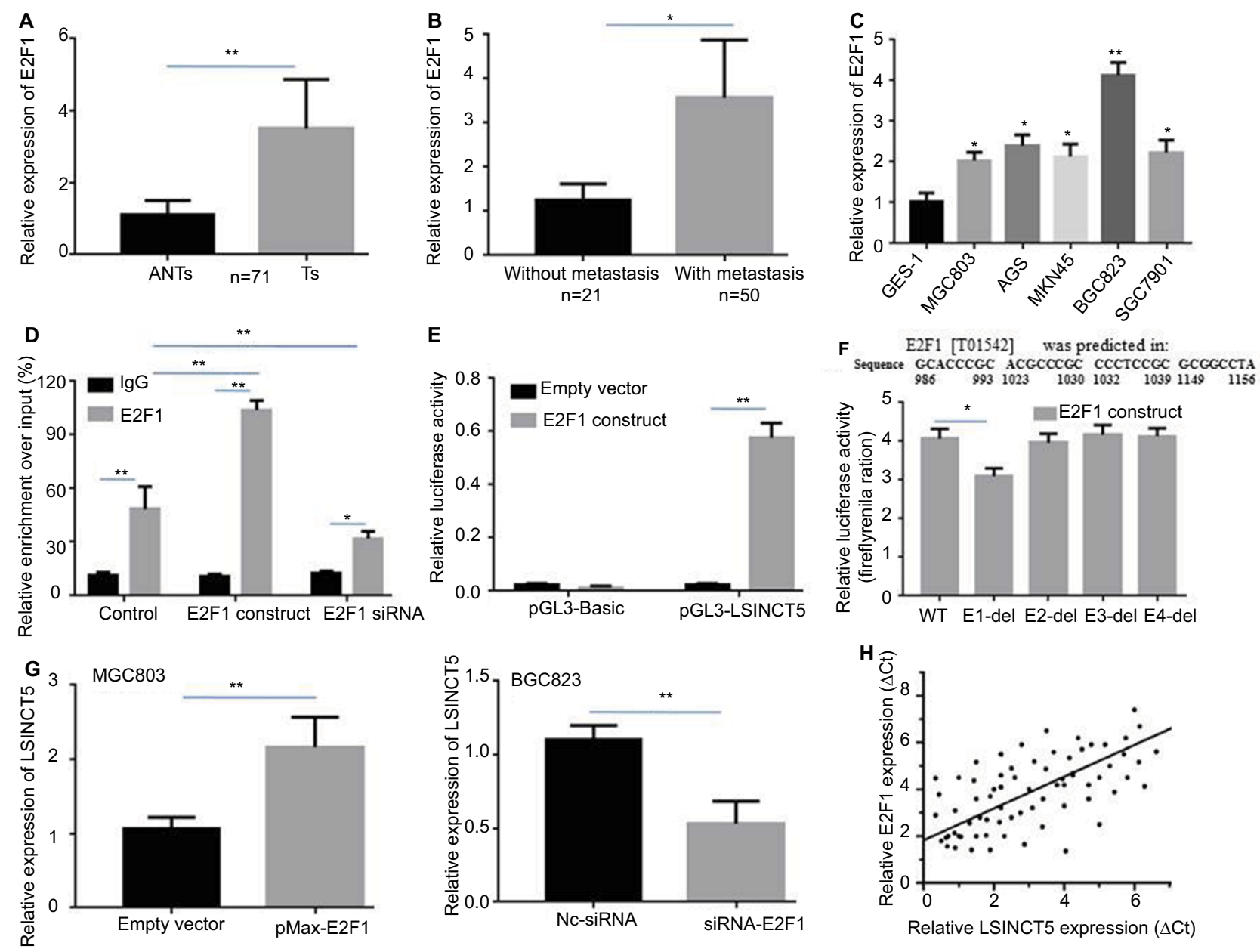

Figure 2 E2FI upregulates LSINCT5 expression in GC cells.

Notes: qRT-PCR analysis of the E2FI expression levels in GC tissues and paired ANTs (A) and in metastatic and nonmetastatic GC tissues (B). (C) qRT-PCR analysis of E2FI expression in a series of human GC cell lines and a normal gastric epithelial cell line (GES-I). (D) ChIP assays using E2FI antibody demonstrated endogenous E2FI binding to the LSINCT5 gene promoter, and ectopic expression or siRNA knockdown increased or reduced E2FI enrichment at the LSINCT5 promoter, respectively. (E) A dual-luciferase reporter assay was performed through cotransfection of the LSINCT5 promoter fragment (pGL3-LSINCT5) and an E2FI-overexpression construct. (F) We examined the LSINCT5 core promoter region for transcription factor binding sites, and identified four tandem putative E2FI-binding sites. Reporter assays were conducted in cells transfected with various LSINCT5 promoter constructs in which different E2FI-binding elements were deleted (WT, del). Luciferase activity is presented relative to that of the PGL3 vector (a promoter-less vector). (G) qRT-PCR analysis of the LSINCT5 expression levels following the transfection of MGC803 and BGC823 cells with a pMax-E2FI expression vector and siRNA-E2FI, respectively. $(\mathbf{H})$ Analysis of the relationship between LSINCT5 and E2FI mRNA levels ( $\triangle$ Ct value) in GC tissues. Bars: SD; $* P<0.05$ and $* * P<0.01$.

Abbreviations: ANTs, adjacent normal tissues; Ts, tumor tissues; ChIP, chromatin immunoprecipitation; del, deletion; GC, gastric cancer; qRT-PCR, quantitative real-time polymerase chain reaction; WT, wild type; NC, negative control. 
at the following regions: $-1,156$ to $-1,149 \mathrm{bp}(\mathrm{E} 1),-1,039$ to $-1,032$ bp (E2), $-1,030$ to $-1,023$ bp (E3) and -993 to -986 bp (E4) (Figure 2F). We subsequently deleted the putative binding sites individually and performed additional reporter assays to validate this aforementioned finding. Deletion of the E1-binding motif significantly impaired the ability of E2F1 to activate LSINCT5 transcription, suggesting that special binding motifs are required for the E2F1-mediated regulation of LSINCT5 transcription (Figure 2F).

We then determined whether E2F1 can mediate LSINCT5 overexpression through both gain- and loss-of-function approaches. The results showed that the ectopic expression or siRNA knockdown of E2F1 increased or reduced E2F1 enrichment to the LSINCT5 promoter, respectively (Figure 2D), and up- or downregulated LSINCT5, respectively, in GC cells (Figure 2G). In addition, the correlation between E2F1 and LSINCT5 gene transcription in GC tissue samples was assessed, and the results revealed that LSINCT5 expression was positively correlated with E2F1 mRNA expression (Pearson $R=0.685, P<0.001$ ) (Figure $2 \mathrm{H}$ ). These results suggest that E2F1 is a transcription factor that activates LSINCT5 transcription and upregulates its expression.

\section{LSINCT5 impacts the EMT in GC cells}

EMT has been shown to be critically important in the early events of tumor cell metastatic dissemination by inducing cell motility and allowing cells to acquire invasion potential. We hypothesized that LSINCT5 influences the malignant phenotype, partly by regulating the EMT. qRT-PCR and Western blotting assays were performed to detect the expression of EMT markers in cells in which LSINCT5 was overexpressed or knocked down. Our results revealed that the upregulation of LSINCT5 expression led to increased N-cadherin, vimentin, and MMP-2 expression and reduced E-cadherin expression (Figure 3A). In contrast, the downregulation of LSINCT5 expression induced E-cadherin expression and decreased N-cadherin, vimentin, MMP-2, and MMP-9 expression (Figure 3B). Moreover, the results of a Western blotting analysis showed that LSINCT5 downregulation stimulated the E-cadherin protein expression and reduced $\mathrm{N}$-cadherin, vimentin, and MMP-2 protein expression in GC cells, whereas LSINCT5 upregulation yielded the opposite effects (Figure 3C). These results support our hypothesis that LSINCT5 influences the malignant cell phenotype by regulating the EMT.

\section{Discussion}

Accumulating evidence has shown that lncRNAs play important roles in cancer pathophysiology and could provide a new avenue for elucidating the biology of this disease. ${ }^{20} \mathrm{Xu}$ et al revealed that the FENDRR inhibits the metastatic behavior of GC cells. ${ }^{21}$ The lncRNA TINCR has been reported to promote GC proliferation by accelerating KLF2 mRNA degradation, ${ }^{22}$ and we previously demonstrated that TUSC 7 is a p53-regulated tumor suppressor that acts in part by repressing miR-23b in GC. ${ }^{23}$ Therefore, the identification of GCassociated lncRNAs may provide missing information related to the well-known oncogenic and tumor suppressor network.

In our previous study, we primarily focused on the clinical significance and cell proliferation function of aberrant LSINCT5 expression. ${ }^{9}$ Because high LSINCT5 expression is associated with a metastatic tumor phenotype, we presumed

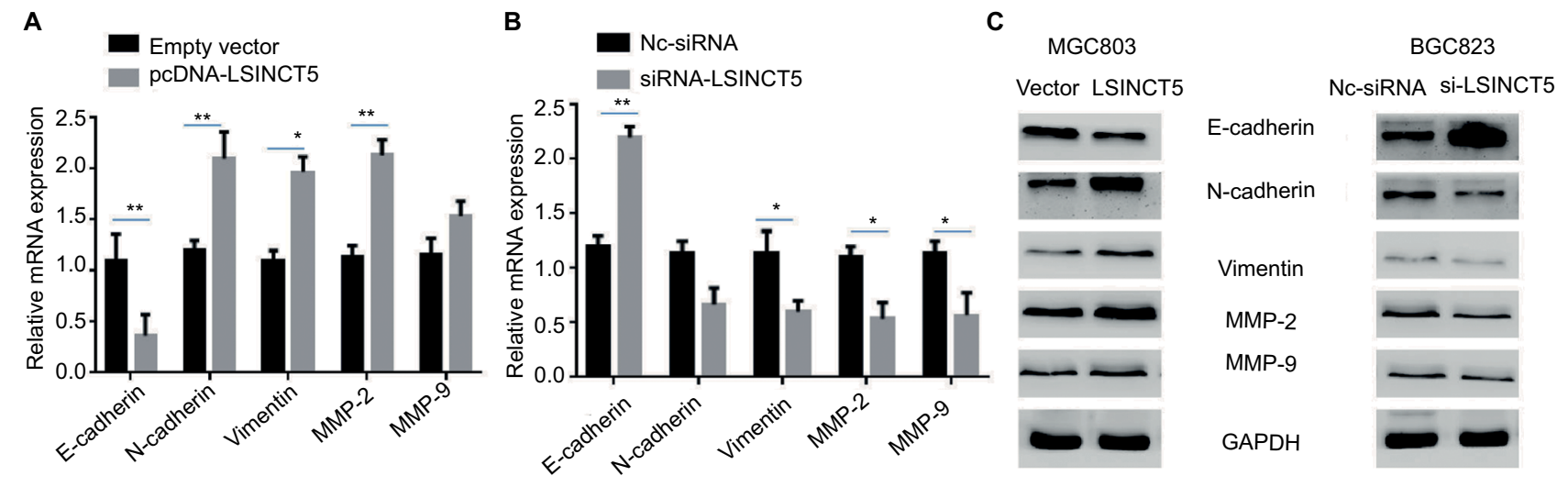

Figure 3 Overexpression of LSINCT5 promotes GC cell invasion and metastasis by affecting the EMT.

Notes: (A, B) qRT-PCR analysis of E-cadherin, N-cadherin, vimentin, MMP-2, and MMP-9 expression following the transfection of MGC803 cells with empty vector or PCDNA-LSINCT5 or the transfection of BGC823 cells with scrambled siRNA or si-LSINCT5. (C) Western blot analysis of E-cadherin, N-cadherin, vimentin, MMP-2, and MMP-9 expression in MGC803 cells transfected with PCDNA-LSINCT5 and BGC823 cells transfected with si-LSINCT5. All the experiments were performed in triplicate. Bars: $S D ; * P<0.05$ and ${ }^{*} * P<0.01$.

Abbreviations: EMT, epithelial-mesenchymal transition; GC, gastric cancer; MMP, matrix metalloproteinase; qRT-PCR, quantitative real-time polymerase chain reaction; NC, negative control. 
that LSINCT5 plays a significant role in the metastasis process. In the current study, we initially revealed that LSINCT5 was overexpressed in metastatic GC tissues. Functional in vitro studies indicated that ectopic LSINCT5 expression significantly promoted cell migration and invasion, whereas the knockdown of LSINCT5 expression impeded these biological processes. These findings indicate that LSINCT5 exerts a marked influence on the progression and metastasis of GC cells and might be a novel metastatic marker for GC.

Previous findings have suggested that E2F protein family members play important roles during the development of human malignancies. ${ }^{15}$ E2F1, which has been reported to be upregulated in $\mathrm{GC},{ }^{19}$ is a key regulator of the $\mathrm{G} 1 / \mathrm{S}$ phase transition, ${ }^{24}$ but its functional role in $\mathrm{GC}$ progression remains debated. In the current study, we found that E2F1 expression was significantly higher in GC tissues compared with corresponding noncancerous tissues. Specifically, E2F1 expression was significantly higher in metastatic tumor tissues than in nonmetastatic tissues. Our results indicate that E2F1 expression may have significant predictive value for the early detection of metastasis in patients with GC.

To explore the mechanism underlying the induction of high LSINCT5 expression, we performed a computational analysis of the LSINCT5 promoter region using several computational algorithms. Because E2F1 plays pivotal roles in tumor development by modulating the expression of both coding and noncoding transcripts, ${ }^{13,14}$ we focused on the prediction of E2F1-binding sites in the LSINCT5 promoter region. A ChIP combined with luciferase reporter assay analysis indicated that E2F1 bound to approximately $-1,156$ to $-1,149$ bp in the LSINCT5 promoter region and specifically activated LSINCT5 transcription. These results indicate that LSINCT5 may be a downstream effector of E2F1 that can control GC cell migration and invasion.

The EMT plays crucial roles in cancer initiation, progression, and particularly metastasis. ${ }^{3,25,26}$ Accumulating data have revealed that lncRNAs regulate tumor metastasis by affecting the EMT. ${ }^{27,28}$ The hallmarks of the EMT are a loss of E-cadherin expression and aberrant $\mathrm{N}$-cadherin and vimentin expression. It is well known that MMPs play key roles in the invasion and metastasis of many types of carcinomas. To explore the potential mechanism through which LSINCT5 contributes to GC invasion and metastasis, we determined the levels of metastasis markers following LSINCT5 overexpression or downregulation. The upregulation of LSINCT5 expression reduced E-cadherin expression and increased N-cadherin, vimentin, and MMP-2 expression, whereas the downregulation of LSINCT5 increased
E-cadherin expression and decreased $\mathrm{N}$-cadherin, vimentin, MMP-2, and MMP-9 expression. Our results indicate that LSINCT5 mediates increases in the migration and invasion of GC cells and possibly affects the EMT. The EMT, a central differentiation process, allows tissue remodeling during the early stages of embryogenesis and is also implicated in the promotion of cancer cell migration and tumor metastasis. Thus, as important and extensive regulators of the EMT, lncRNAs might be suitable candidates for cancer treatment. Recent years have shown progress in molecular therapeutics targeting key drivers of cancer progression, ${ }^{29}$ and LSINCT5, an important regulator of the EMT, shows promise as a drug target. Drugs that regulate LSINCT5 expression have the potential to be used for clinical application, and clinical tests or assays should be developed to test such therapeutics.

\section{Conclusion}

In summary, our results showed that LSINCT5 is markedly upregulated in metastatic GC tissues. Moreover, upregulated LSINCT5 may be a downstream effector of E2F1 that promotes $\mathrm{GC}$ cell migration and invasion by affecting the EMT. However, the molecular mechanisms through which LSINCT5 regulates the EMT require further investigation.

\section{Acknowledgments}

The work was supported by the National Natural Science Foundation of China (81502429, 81472220, 81470353), the Science and Technology Commission of the Shanghai Municipality (15495810300), the Shanghai Hospital Development Center Emerging Advanced Technology Joint Research Project (SHDC12014105), the Shanghai Key Developing Disciplines (2015ZB0201), the Shanghai Science and Technology Development Fund (No. 15ZR1407400), and Shanghai Municipal Commission of Health and Family Planning (2015ZB0204).

\section{Disclosure}

The authors report no conflicts of interest in this work.

\section{References}

1. Torre LA, Bray F, Siegel RL, et al. Global cancer statistics, 2012. CA Cancer J Clin. 2015;65(2):87-108.

2. Patru CL, Surlin V, Georgescu I, Patru E. Current issues in gastric cancer epidemiology. Rev Med Chir Soc Med Nat Iasi. 2013;117(1):199-204.

3. Thiery JP, Acloque H, Huang RY, Nieto MA. Epithelial-mesenchymal transitions in development and disease. Cell. 2009;139(5):871-890.

4. Qi P, du X. The long non-coding RNAs, a new cancer diagnostic and therapeutic gold mine. Mod Pathol. 2013;26(2):155-165.

5. Shen XH, Qi P, Du X. Long non-coding RNAs in cancer invasion and metastasis. Mod Pathol. 2015;28(1):4-13. 
6. Xu MD, Wang Y, Weng W, et al. A Positive Feedback Loop of lncRNAPVT1 and FOXM1 Facilitates Gastric Cancer Growth and Invasion. Clin Cancer Res. 2017;23(8):2071-2080.

7. Guil S, Esteller M. Cis-acting noncoding RNAs: friends and foes. Nat Struct Mol Biol. 2012;19(11):1068-1075.

8. Silva JM, Boczek NJ, Berres MW, Ma X, Smith DI. LSINCT5 is over expressed in breast and ovarian cancer and affects cellular proliferation. RNA Biol. 2011;8(3):496-505.

9. Xu MD, Qi P, Weng WW, et al. Long non-coding RNA LSINCT5 predicts negative prognosis and exhibits oncogenic activity in gastric cancer. Medicine. 2014;93(28):e303.

10. Chen HZ, Tsai SY, Leone G. Emerging roles of E2Fs in cancer: an exit from cell cycle control. Nat Rev Cancer. 2009;9(11):785-797.

11. Burkhart DL, Sage J. Cellular mechanisms of tumour suppression by the retinoblastoma gene. Nat Rev Cancer. 2008;8(9):671-682.

12. Bertoli C, Skotheim JM, de Bruin RA. Control of cell cycle transcription during G1 and S phases. Nat Rev Mol Cell Biol. 2013;14(8):518-528.

13. Tarangelo A, Lo N, Teng R, et al. Recruitment of Pontin/Reptin by E2f1 amplifies E2f transcriptional response during cancer progression. Nat Commun. 2015;6:10028.

14. Sun M, Nie F, Wang Y, et al. LncRNA HOXA11-AS Promotes Proliferation and Invasion of Gastric Cancer by Scaffolding the Chromatin Modification Factors PRC2, LSD1, and DNMT1. Cancer Res. 2016;76(21):6299-6310.

15. Engelmann D, Pützer BM. The dark side of E2F1: in transit beyond apoptosis. Cancer Res. 2012;72(3):571-575.

16. Yamasaki L, Bronson R, Williams BO, Dyson NJ, Harlow E, Jacks T. Loss of E2F-1 reduces tumorigenesis and extends the lifespan of Rb1(+/-)mice. Nat Genet. 1998;18(4):360-364.

17. Xie Y, Wang C, Li L, Ma Y, Yin Y, Xiao Q. Overexpression of E2F-1 inhibits progression of gastric cancer in vitro. Cell Biol Int. 2009;33(6):640-649.

18. Xie Y, Yin Y, Li L, Ma Y, Xiao Q. Short interfering RNA directed against the E2F-1 gene suppressing gastric cancer progression in vitro. Oncol Rep. 2009;21(5):1345-1353.
19. Zhang X, Ni Z, Duan Z, et al. Overexpression of E2F mRNAs associated with gastric cancer progression identified by the transcription factor and miRNA co-regulatory network analysis. PLoS One. 2015;10(2):e0116979.

20. Xu MD, Qi P, du X. Long non-coding RNAs in colorectal cancer: implications for pathogenesis and clinical application. Mod Pathol. 2014;27(10):1310-1320.

21. Xu TP, Huang MD, Xia R, et al. Decreased expression of the long noncoding RNA FENDRR is associated with poor prognosis in gastric cancer and FENDRR regulates gastric cancer cell metastasis by affecting fibronectin1 expression. J Hematol Oncol. 2014;7:63.

22. Xu TP, Liu XX, Xia R, et al. SP1-induced upregulation of the long noncoding RNA TINCR regulates cell proliferation and apoptosis by affecting KLF2 mRNA stability in gastric cancer. Oncogene. 2015;34(45):5648-5661.

23. Qi P, Xu MD, Shen XH, et al. Reciprocal repression between TUSC7 and miR-23b in gastric cancer. Int J Cancer. 2015;137(6):1269-1278.

24. Hallstrom TC, Nevins JR. Balancing the decision of cell proliferation and cell fate. Cell Cycle. 2009;8(4):532-535.

25. de Craene B, Berx G. Regulatory networks defining EMT during cancer initiation and progression. Nat Rev Cancer. 2013;13(2):97-110.

26. Guo F, Parker Kerrigan BC, Yang D, et al. Post-transcriptional regulatory network of epithelial-to-mesenchymal and mesenchymal-to-epithelial transitions. J Hematol Oncol. 2014;7:19.

27. Yuan JH, Yang F, Wang F, et al. A long noncoding RNA activated by TGF- $\beta$ promotes the invasion-metastasis cascade in hepatocellular carcinoma. Cancer Cell. 2014;25(5):666-681.

28. Liu F, Yuan JH, Huang JF, et al. Long noncoding RNA FTX inhibits hepatocellular carcinoma proliferation and metastasis by binding MCM2 and miR-374a. Oncogene. 2016;35(41):5422-5434.

29. Liu LK, Jiang XY, Zhou XX, Wang DM, Song XL, Jiang HB. Upregulation of vimentin and aberrant expression of E-cadherin/beta-catenin complex in oral squamous cell carcinomas: correlation with the clinicopathological features and patient outcome. Mod Pathol. 2010;23(2): 213-224. 


\section{Supplementary material}

Table SI Primers used in this study

Qualitative real-time polymerase chain reaction primers

LSINCT5: 5'-TTCGGCAAGCTCCTTTTCTA-3' (forward) and

5'-GCCCAAGTCCCAAAAAGTTCT-3' (reverse);

GAPDH: 5'-CCGGGAAACTGTGGCGTGATGG-3' (forward) and

5'-AGGTGGAGGAGTGGGTGTCGCTGTT-3' (reverse);

E2FI: 5'-TGCAGAGCAGATGGTTATGG-3' (forward) and

5'-AGATGATGGTGGTGGTGACA-3' (reverse).

Cancer Management and Research

\section{Publish your work in this journal}

Cancer Management and Research is an international, peer-reviewed open access journal focusing on cancer research and the optimal use of preventative and integrated treatment interventions to achieve improve outcomes, enhanced survival and quality of life for the cancer patient The manuscript management system is completely online and includes 